\title{
SIGNAL RECONSTRUCTION FROM MULTIPLE UNREGISTERED SETS OF SAMPLES USING GROEBNER BASES
}

\author{
Patrick Vandewalle ${ }^{\ddagger}$, Luciano Sbaiz ${ }^{\ddagger}$ and Martin Vetterli $\ddagger \S$ \\ $\ddagger$ LCAV - School of Computer and Communication Sciences \\ Ecole Polytechnique Fédérale de Lausanne (EPFL), CH-1015 Lausanne, Switzerland \\ $\S$ Department of Electrical Engineering and Computer Science \\ UC Berkeley, Berkeley CA94720, USA
}

\begin{abstract}
We present a new method for signal reconstruction from multiple sets of samples with unknown offsets. We rewrite the reconstruction problem as a set of polynomial equations in the unknown signal parameters and the offsets between the sets of samples. Then, we construct a Gröbner basis for the corresponding affine variety. The signal parameters can then easily be derived from this Gröbner basis. This provides us with an elegant solution method for the initial nonlinear problem. We show two examples for the reconstruction of polynomial signals and Fourier series.
\end{abstract}

\section{INTRODUCTION}

In this paper, we present a method to reconstruct a signal from multiple sets of samples using Gröbner bases. The offsets between the different sets of samples are unknown, and can take any real value. Each of the individual sets of samples is sampled uniformly, at a rate below the Nyquist rate. This is the typical setup that is used in super-resolution imaging, where a high resolution, aliasing-free image is reconstructed from a set of low resolution, aliased images with small relative shifts [1]. Similarly, in time-interleaved A/D converters multiple low-rate converters are combined to build the equivalent of a high-rate converter [2]. One of the important issues in this domain is the synchronization of the low-rate converters.

The reconstruction problem from multiple sets of samples with known offsets was solved by Papoulis [3] for bandlimited signals. Marziliano et al. [4] describe a reconstruction method for multiple sets of samples with unknown, but discrete-valued offsets using a combinatorial method. Subspace-based methods for the reconstruction with unknown real-valued offsets between the sets of samples are presented by Vandewalle et al. [5, 6].

The work presented in this paper was supported by the National Competence Center in Research on Mobile Information and Communication Systems (NCCR-MICS, http://www.mics.org), a center supported by the Swiss National Science Foundation under grant number 5005-67322.

\section{PROBLEM SETUP}

Consider a signal $f(t)$ that belongs to the finite-dimensional space spanned by a basis $\mathcal{B}=\left\{\phi_{l}(t)\right\}_{l=0 . . L-1}$. The signal $f(t)$ can then be written in this basis as

$$
f(t)=\sum_{l=0}^{L-1} x_{l} \phi_{l}(t),
$$

where $x_{l}$ is the expansion coefficient corresponding to $\phi_{l}(t)$. The function $f(t)$ is sampled using $M$ sets of $N$ regularly spaced samples. The offsets between the sets of samples are called $t_{m}\left(0 \leq m<M\right.$, with $\left.t_{0}=0\right)$. The $m$-th set of samples can be written in a vector as

$$
\mathbf{y}_{m}=\boldsymbol{\Phi}_{t_{m}} \mathbf{x}
$$

with $\boldsymbol{\Phi}_{t_{m}}$ the $N \times L$ matrix containing the $L$ basis functions $\phi_{l}(t)$ sampled uniformly with offset $t_{m}\left(\boldsymbol{\Phi}_{t_{m}}(i, j)=\right.$ $\left.\phi_{j}\left(i / N+t_{m}\right)\right)$, and $\mathbf{x}$ the expansion vector. The different sample vectors $\mathbf{y}_{m}$ can then be combined into a large vector $\mathbf{y}$ (and similarly for $\boldsymbol{\Phi}_{\mathbf{t}}$ ):

$$
\mathbf{y}=\Phi_{\mathbf{t}} \mathbf{x}
$$

where both the expansion coefficients $\mathbf{x}$ and the offsets $\mathbf{t}$ (which appear in the matrix $\boldsymbol{\Phi}_{\mathbf{t}}$ ) are unknown. This setup is illustrated in Figure 1.

\section{GRÖBNER BASES}

Gröbner bases are commonly used in algebraic geometry for systems of polynomial equations in one or more variables. An important property of Gröbner bases is that they always lead to a description of all the possible solutions for the system of equations. A complete presentation of this domain is out of the scope of this paper, but can be found in Cox et al. [7]. We will concentrate on the main ideas that are of importance for our signal reconstruction problem. Next to super-resolution, Gröbner basis techniques could potentially also give elegant solutions to other signal processing problems. 


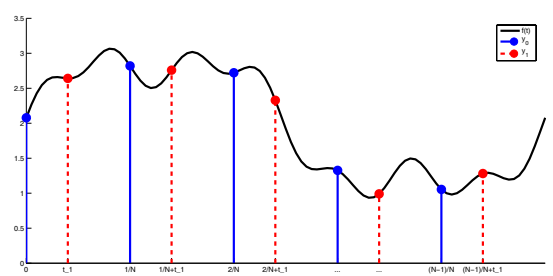

(a)

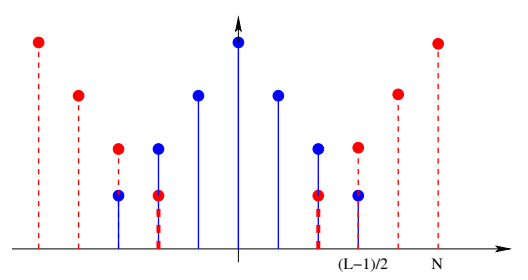

(b)

Fig. 1. Illustration of the different variables from Section 2 for $M=2$ using a Fourier basis. (a) Time domain representation of the signal $f(t)$ and its sets of samples $\mathbf{y}_{0}$ and $\mathbf{y}_{1}$. (b) Frequency domain representation of the absolute value of the signal $f(t)$ and its aliased versions after sampling.

The central objects in algebraic geometry are affine varieties. An affine variety $V$ is defined as the set of all the common zeros of a set of polynomial equations in one or more variables $x_{1}, \ldots, x_{n}$ :

$$
V=\left\{\mathbf{x}=\left(x_{0}, x_{1}, \ldots, x_{n-1}\right) \mid p_{0}(\mathbf{x})=p_{1}(\mathbf{x})=\ldots=0\right\},
$$

where each $p_{i}(\mathbf{x})$ is a polynomial in the variables $\mathbf{x}=$ $\left(\begin{array}{lll}x_{0} & \ldots & x_{n-1}\end{array}\right)$. In our case, for a polynomial basis $\mathcal{B}$, the variety is defined as the set of solutions for $\boldsymbol{\Phi}_{\mathbf{t}} \mathbf{x}-\mathbf{y}=0$. We will see in the examples in Section 4 and 5 that if there are enough samples and sampling sets (and therefore equations), the variety contains a single point, and the solution of the reconstruction problem is unique.

In the simpler case of a linear affine variety, the set of solutions can be computed using Gaussian elimination. Recall that the Gaussian elimination algorithm essentially consists in computing linear combinations of the equations to progressively eliminate all the variables.

For an arbitrary affine variety, the computation of a Gröbner basis can be seen as a generalization of Gaussian elimination to polynomials. As in Gaussian elimination, we will try to replace the initial set of equations by equivalent sets (defining the same variety), from which the unknowns can be derived more easily. Instead of the scalar coefficients in the linear combinations with Gaussian elimination, we will now use polynomial coefficients to combine different equations. Just as in the linear case, we need to fix an ordering for the terms of the polynomial. A common choice is lexicographic ordering.

Gröbner bases are defined for ideals. An ideal can be de- fined as the set of all the polynomials that can be generated as linear combinations of a given set of polynomials. Again, polynomial coefficients can be used in these linear combinations. For each affine variety, a corresponding ideal can be defined from the polynomials of the variety. The ideal corresponding to the variety $V$ given in (4) can be written as

$$
I_{V}=\left\{p \mid p=\sum_{i} a_{i}(\mathbf{x}) p_{i}(\mathbf{x})\right\} .
$$

The coefficients $a_{i}(\mathbf{x})$ are again polynomials in $x_{0}, \ldots, x_{n-1}$.

To determine whether a given polynomial belongs to an ideal $I$, we need to check if it can be written as a linear combination of the polynomials in the ideal. In Gaussian elimination, we used Euclid's algorithm for the greatest common divisor to do this. With polynomials in multiple variables, this is not possible, because they do not form a Euclidean domain. However, we can define a multivariate division, allowing us to compute the division of one multivariate polynomial by another polynomial, and its remainder. Unfortunately, the division of a polynomial by a set of polynomials may depend on the order in which the polynomials are given.

Now that we have ideals and a method for polynomial division, we can define a Gröbner basis. A set of polynomials $G=\left\{g_{0}, \ldots, g_{k}\right\}$ forms a Gröbner basis for the ideal $I$ if the division of any polynomial $p \in I$ by the polynomials of $G$ has remainder zero. Moreover, for a Gröbner basis, the result of the division does not depend on the order in which the polynomials $g_{i}$ are given.

Since the polynomials form a ring, it is not always possible to compute the inverse of the (polynomial) coefficients used in linear combinations. We can therefore not always replace a polynomial by its linear combination with another polynomial, as we did in Gaussian elimination. If we would do this, it is possible that we cannot reconstruct the original polynomial, and thus we modify the variety. Therefore, it is necessary to expand the set of polynomials first, until a Gröbner basis is obtained. Afterwards, some of the polynomials may be removed, if the remaining ones are sufficient to generate the ideal and still form a Gröbner basis.

The first algorithm to compute a Gröbner basis in this way was developed by Buchberger. It can be shown that this algorithm always finds the (unique) reduced Gröbner basis for a specific ordering. Many improvements on this algorithm have been proposed, for example by Faugère [8].

A very important property for solving equations using Gröbner bases is given by the elimination theorem. Assume we have an ideal $I$ with Gröbner basis $G$, and we use lexicographic ordering. The elimination theorem states that a Gröbner basis for the polynomials of $I$ in only the variables $x_{k}, \ldots, x_{n-1}$ is then given by the polynomials of $G$ that only depend on $x_{k}, \ldots, x_{n-1}$. In other words, a set of polynomial equations can be solved from its Gröbner basis using backsubstitution, just like we did with Gaussian elimination. At least one variable is eliminated in every iteration. The ideals 
$I_{k}$ formed by intersecting the ideal $I$ with decreasing numbers of variables $x_{k}, \ldots, x_{n-1}$ are called elimination ideals.

\section{POLYNOMIAL RECONSTRUCTION}

We can apply Buchberger's algorithm to our signal reconstruction problem. The first step is to write the equations of the system (3) as a system of polynomial equations. The simplest case is when the basis $\mathcal{B}$ is a polynomial basis. For such a basis, the equations from (3) are already in polynomial form, and all we need to do is to compute a Gröbner basis for the corresponding ideal. The signal parameters can then directly be derived from the basis. We illustrate this with an example. Example: Consider the case where the basis $\mathcal{B}$ is given by the functions $\phi_{l}(t)=t^{l}, l=0, \ldots, L-1$ with $L=3$. Assume that we take two sets of two samples, i.e. $N=2, M=2$. Consider the signal parameter vector $\mathbf{x}=\left(\begin{array}{lll}64 & -24 & -4\end{array}\right)^{T}$ and the displacements $\mathbf{t}=$ $\left(\begin{array}{ll}0 & 1 / 8\end{array}\right)^{T}$. In this case, the two sets of measurements would be $y_{0}=\left(\begin{array}{cc}-4 & 0\end{array}\right)^{T}$ and $y_{1}=\left(\begin{array}{ll}-6 & 6\end{array}\right)^{T}$. Therefore, the system that we want to solve is

$$
\left(\begin{array}{ccc}
0 & 0 & 1 \\
\frac{1}{4} & \frac{1}{2} & 1 \\
t^{2} & t & 1 \\
\left(\frac{1}{2}+t\right)^{2} & \frac{1}{2}+t & 1
\end{array}\right)\left(\begin{array}{l}
x_{0} \\
x_{1} \\
x_{2}
\end{array}\right)=\left(\begin{array}{c}
-4 \\
0 \\
-6 \\
6
\end{array}\right)
$$

We can represent the set of solutions of (6) as the points of the affine variety defined by the set of polynomials:

$$
\begin{aligned}
& p_{0}=x_{2}+4 \\
& p_{1}=\frac{1}{4} x_{0}+\frac{1}{2} x_{1}+x_{2} \\
& p_{2}=x_{0} t^{2}+x_{1} t+x_{2}+6 \\
& p_{3}=x_{0} t^{2}+x_{0} t+\frac{1}{4} x_{0}+x_{1} t+\frac{1}{2} x_{1}+x_{2}-6
\end{aligned}
$$

in the variables $x_{0}, x_{1}, x_{2}$ and $t$. We fix the ordering of variables as $x_{0}>x_{1}>x_{2}>t$ and we use lexicographic ordering for monomials.

At the first step of Buchberger's algorithm, we add the following polynomials to the basis:

$$
\begin{aligned}
& p_{4}=-8 x_{1} t^{2}+4 x_{1} t+64 t^{2}+8 \\
& p_{5}=-8 x_{1} t^{2}-4 x_{1} t+64 t^{2}+64 t-24 \\
& p_{6}=2 x_{1} t-16 t+8
\end{aligned}
$$

Following the same procedure, in the second iteration, we find that only two new non-zero polynomials can be computed. We add

$$
\begin{aligned}
& p_{7}=-2 x_{1}-48 \\
& p_{8}=64 t-8
\end{aligned}
$$

to the basis. In the following iteration all remainders are zero and we conclude that $p_{0}, \ldots, p_{8}$ is a Gröbner basis. We can now try to reduce the elements of the basis. In this case, we have that $p_{2}, p_{3}, p_{4}, p_{5}, p_{6}$ can be removed and the final basis is given by $\left\{p_{0}, p_{1}, p_{7}, p_{8}\right\}$. In order to apply the elimination theorem, we rename the elements of the basis as:

$$
\begin{aligned}
& g_{0}=\frac{1}{4} x_{0}+\frac{1}{2} x_{1}+x_{2} \\
& g_{1}=-2 x_{1}-48 \\
& g_{2}=x_{2}+4 \\
& g_{3}=64 t-8 .
\end{aligned}
$$

The elimination ideals are $I_{0}=\left\langle g_{1}, g_{2}, g_{3}\right\rangle, I_{1}=\left\langle g_{2}, g_{3}\right\rangle$, and $I_{2}=\left\langle g_{3}\right\rangle$. The solution of the problem can be obtained by computing the points of the affine variety associated to $I_{2}$ and extending it by back substitution to $I_{1}, I_{0}$ and $I$. We easily find that the unique solution is $t=1 / 8, x_{2}=-4$, $x_{1}=-24$, and $x_{0}=64$.

\section{SIGNAL RECONSTRUCTION IN OTHER BASES}

The procedure described in the example above can be applied to any polynomial basis. More generally, using a change of variable, we can solve the problem when the basis is a set of functions $\phi_{l}(t)=h(t)^{l}$, with $h(t)$ an invertible function. An important case is when $h(t)=e^{2 \pi j t}$, which gives the Fourier series. In fact, consider the case of a complex signal of the form

$$
f(t)=\sum_{l=-L+1}^{L-1} x_{l} \phi_{l}(t)
$$

with $\phi_{l}(t)=e^{2 \pi j l t}$. The samples are given by

$$
y_{m}(n)=f\left(\frac{n}{N}+t_{m}\right)=\sum_{l=-L+1}^{L-1} x_{l} W_{N}^{n l} e^{2 \pi j l t_{m}}
$$

with $W_{N}=e^{2 \pi j / N}$. By setting, $z_{m}=e^{2 \pi j t_{m}}$, each of the samples gives a polynomial constraint of the form:

$$
p_{n N+m}=\sum_{l=-L+1}^{L-1} x_{l} W_{N}^{n l} z_{m}^{l+L-1}-z_{m}^{L-1} y_{m}(n)=0 .
$$

As in the previous case, the solution of the problem is given by the points that belong to an affine variety. We can therefore again compute the signal parameters from a Gröbner basis for this variety. We illustrate this with an example for the Fourier basis.

Example: Assume $L=2$, i.e. the input signal is represented by the parameter vector $\mathbf{x}=\left(x_{-2}, \ldots, x_{2}\right)$, where each entry is a complex value. For this example, we assume

$$
\mathbf{x}=\left(\begin{array}{lllll}
3 & 2-j & 1 & 2+j & 3
\end{array}\right)^{T} .
$$

We suppose that $M=2$ sets of $N=4$ samples are taken from the input signal, with the displacements $\mathbf{t}=\left(\begin{array}{ll}0 & 1 / 8\end{array}\right)^{T}$. In this case, the two sets of measurements are

$$
\begin{aligned}
& y_{0}=\left(\begin{array}{llll}
11 & -7 & 3 & -3
\end{array}\right)^{T} \\
& y_{1}=\left(\begin{array}{llll}
1+\sqrt{2} & 1-3 \sqrt{2} & 1-\sqrt{2} & 1+3 \sqrt{2}
\end{array}\right)^{T} .
\end{aligned}
$$


Applying (13), we obtain 8 polynomials that represent the constraints imposed by the measurements:

$$
\begin{aligned}
p_{0}= & x_{2}+x_{1}+x_{0}+x_{-1}+x_{-2}-11 \\
p_{1}= & -x_{2}+j x_{1}+x_{0}-j x_{-1}-x_{-2}+7 \\
p_{2}= & x_{2}-x_{1}+x_{0}-x_{-1}+x_{-2}-3 \\
p_{3}= & -x_{2}-j x_{1}+x_{0}+j x_{-1}-x_{-2}+3 \\
p_{4}= & x_{2} z_{1}^{4}+x_{1} z_{1}^{3}+x_{0} z_{1}^{2}+x_{-1} z_{1}+x_{-2}-(1+\sqrt{2}) z_{1}^{2} \\
p_{5}= & -x_{2} z_{1}^{4}+j x_{1} z_{1}^{3}+x_{0} z_{1}^{2}-j x_{-1} z_{1}-x_{-2} \\
& -(1-3 \sqrt{2}) z_{1}^{2} \\
p_{6}= & x_{2} z_{1}^{4}-x_{1} z_{1}^{3}+x_{0} z_{1}^{2}-x_{-1} z_{1}+x_{-2}-(1-\sqrt{2}) z_{1}^{2} \\
p_{7}= & -x_{2} z_{1}^{4}-j x_{1} z_{1}^{3}+x_{0} z_{1}^{2}+j x_{-1} z_{1}-x_{-2} \\
& -(1+3 \sqrt{2}) z_{1}^{2},
\end{aligned}
$$

where the complex variable $z_{1}=e^{j 2 \pi t_{1}}$ represents the displacement. Again, we can compute a Gröbner basis using Buchberger's algorithm. Assuming the ordering $x_{2}>x_{1}>$ $\ldots>x_{-2}>z_{1}$, we obtain

$$
\begin{aligned}
& g_{0}=2 x_{2}-3 j \sqrt{2} z_{1}+3 \sqrt{2} z_{1}-12 \\
& g_{1}=x_{1}-2-j \\
& g_{2}=x_{0}-1 \\
& g_{3}=x_{-1}-2+j \\
& g_{4}=2 x_{-2}+3 j \sqrt{2} z_{1}-3 \sqrt{2} z_{1} \\
& g_{5}=2 z_{1}^{2}-\sqrt{2}(1+j) z_{1} .
\end{aligned}
$$

The last polynomial of the basis, $g_{5}$ eliminates all variables but $z_{1}$. Therefore we can compute the solutions for the displacement variable, $z_{1}=0$ and $z_{1}=e^{j \pi / 4}$. Clearly, $z_{1}=0$ is discarded since it does not belong to the unit circle, while the second solution corresponds to the correct displacement $t_{1}=1 / 8$. By backsubstitution, one can compute the signal parameters.

From the above examples, we can see that this method can be applied to any reconstruction problem that can be written in the form of (3), with polynomial functions of the offsets $t$ in $\Phi_{t}$.

\section{COMPLEXITY}

The computational complexity of our algorithm depends on the specific polynomials $p_{0}, \ldots, p_{M N-1}$, and thus on the signal to reconstruct $f(t)$. For specific choices of $f(t)$, Buchberger's algorithm will quickly lead to a Gröbner basis, in very few iterations. However, for other signals, Buchberger's algorithm will first introduce a large expansion of the basis before it is complete.

In our case, we can see from (3) that the equations are linear in the $L$ variables $x_{l}$ (the signal expansion coefficients). Higher degrees are only found with the $M$ variables $t_{m}$ (the offsets). Therefore, if we place the expansion coefficients first in our ordering (i.e. $x_{0}<\ldots<x_{L-1}<t_{0}<\ldots<t_{M-1}$ ), most of the variables (the first $L$ ) can be eliminated in $\mathrm{O}\left(L^{2}\right)$ steps, using Gaussian elimination. Buchberger's algorithm only needs to be applied on the remaining equations in the $M$ offset variables.

Note that we need to compute the zeros of a polynomial for the backsubstitution step of our solution method. Depending on the order of the polynomial, this may not always be a trivial operation.

\section{CONCLUSIONS}

We presented a method to reconstruct a signal from multiple sets of samples. The problem is first rewritten as a system of polynomial equations. Next, we compute a Gröbner basis for the corresponding ideal. The signal parameters can then easily be derived from this Gröbner basis. In this way, the nonlinear problem in the joint unknown signal coefficients and offsets is highly simplified. We illustrated our method with examples for the reconstruction of polynomials and bandlimited signals.

\section{REFERENCES}

[1] Sung Cheol Park, Min Kyu Park, and Moon Gi Kang, "Super-resolution image reconstruction: A technical overview," IEEE Signal Processing Magazine, vol. 20, no. 3, pp. 21-36, May 2003.

[2] Tanja C. Hofner, "Boost your sampling rate with timeinterleaved data converters," Sensors Magazine, Feb. 2001.

[3] Athanasios Papoulis, "Generalized sampling expansion," IEEE Transactions on Circuits and Systems, vol. 24, no. 11, pp. 652-654, November 1977.

[4] Pina Marziliano and Martin Vetterli, "Reconstruction of irregularly sampled discrete-time bandlimited signals with unknown sampling locations," IEEE Transactions on Signal Processing, vol. 48, no. 12, pp. 3462-3471, December 2000.

[5] Patrick Vandewalle, Luciano Sbaiz, Joos Vandewalle, and Martin Vetterli, "How to take advantage of aliasing in bandlimited signals," in Proc. IEEE ICASSP, May 2004, vol. 3, pp. 948-951.

[6] Patrick Vandewalle, Luciano Sbaiz, Martin Vetterli, and Sabine Süsstrunk, "Super-resolution from highly undersampled images," in Proc. IEEE ICIP, Sept. 2005, vol. 1, pp. 889-892.

[7] David A. Cox, John B. Little, and Don O'Shea, Ideals, Varieties and Algorithms, Springer-Verlag, second edition, 1996.

[8] J.-C. Faugère, "A new efficient algorithm for computing gröbner bases $\left(f_{4}\right)$, , Journal of Pure and Applied Algebra, vol. 139, no. 1-3, pp. 61-88, 1999. 